\title{
Realistic Rashba and Dresselhaus spin-orbit coupling for neutral atoms
}

\author{
D. L. Campbell, ${ }^{1}$ G. Juzeliūnas, ${ }^{2}$ and I. B. Spielman ${ }^{1}$ \\ ${ }^{1}$ Joint Quantum Institute, National Institute of Standards and Technology, and University of Maryland, Gaithersburg, Maryland 20899, USA \\ ${ }^{2}$ Institute of Theoretical Physics and Astronomy, Vilnius University, A. Goštauto 12, Vilnius LT-01108, Lithuania
}

(Received 19 February 2011; published 30 August 2011)

\begin{abstract}
We describe a new class of atom-laser coupling schemes which lead to spin-orbit-coupled Hamiltonians for ultracold neutral atoms. By properly setting the optical phases, a pair of degenerate pseudospin (a linear combination of internal atomic) states emerge as the lowest-energy eigenstates in the spectrum and are thus immune to collisionally induced decay. These schemes use $N$ cyclically coupled ground or metastable internal states. We focus on two situations: a three-level case and a four-level case, where the latter adds a controllable Dresselhaus contribution. We describe an implementation of the four-level scheme for ${ }^{87} \mathrm{Rb}$ and analyze its sensitivity to typical laboratory noise sources. Last, we argue that the Rashba Hamiltonian applies only in the large intensity limit since any laser coupling scheme will produce terms nonlinear in momentum that decline with intensity.
\end{abstract}

DOI: 10.1103/PhysRevA.84.025602

PACS number(s): 67.85.-d, 32.10.Fn, 33.60.+q, 37.10.Gh

Spin-orbit (SO) coupling is essential for realizing topological insulators, noninteracting fermionic systems with topological order [1,2], and yet in other contexts it leads to parasitic effects such as reduced spin coherence times [3]. As with the progression from the single-particle integer quantum Hall effect (IQHE) to the interaction-driven fractional quantum Hall effects (FQHEs), the next important step is realizing the strongly interacting cousins to the topological insulators, of which topological superconductors are a first example [4,5]. Since ultracold atoms lack intrinsic SO coupling, numerous techniques for generating SO coupling (generally equivalent to non-Abelian gauge potentials [6]) with optical [7-11] and now rf [12] fields have been suggested, one of which was recently implemented [13].

Current proposals for realizing SO coupling suffer from two primary limitations. First, the pair of dressed spin states composing the effective spin- $1 / 2$ system are not the two lowest energy states, so collisional deexcitation [14] can rapidly transfer population into the ground state [15]. Second, the required tripod coupling scheme is difficult to directly realize in alkali atoms [16] In this Brief Report, we introduce a class of laser coupling techniques that overcome these difficulties, and we explore the departure of such models from the ideal case (suitable only at infinite laser intensity).

In condensed matter systems, SO coupling links the linear or crystal (not orbital) momentum $\hbar \mathbf{k}$ to the spin of an electron, for example. For systems confined to two dimensions (2D), terms linear in momentum can be represented as a sum of Rashba $\alpha\left(\check{\sigma}_{x} k_{y}-\check{\sigma}_{y} k_{x}\right)$ and Dresselhaus $\beta\left(\check{\sigma}_{x} k_{y}+\check{\sigma}_{y} k_{x}\right)$ SO couplings $[17,18]$, where $\check{\sigma}_{x, y, z}$ are the Pauli matrices. Proposals for creating SO coupling with neutral atoms use lasers to link states of different momentum and spin. Because these lasers impinge from discrete directions, the system lacks the continuous rotational symmetry of the pure Rashba Hamiltonian anticipated in earlier works $[10,11,19]$. We show how a

Published by the American Physical Society under the terms of the Creative Commons Attribution 3.0 License. Further distribution of this work must maintain attribution to the author(s) and the published article's title, journal citation, and DOI. perturbative treatment restores the system's $N$-fold rotational symmetry and demonstrate that these pure couplings are only exact in the limit of infinite laser-atom coupling strength.

We consider $N$ ground or metastable atomic "spin" states $\{|1\rangle,|2\rangle, \ldots,|N\rangle\}$ cyclicly coupled together with complex valued matrix elements $\Omega_{j+1, j}=-\Omega \exp \left[i\left(\mathbf{k}_{j} \cdot \mathbf{x}+\gamma_{j}\right)\right]$ representing plane waves that link consecutive states $|j\rangle$ to $|j+1\rangle$. Here, $\Omega$ describes the optical coupling strength; $\hbar \mathbf{k}_{j}$ and $\gamma_{j}$ are the respective discreet momentum and phase acquired in the $j \rightarrow j+1$ transition. Throughout this manuscript we apply "periodic boundary conditions" $|N+1\rangle=|1\rangle$ for spin states. See, for example, the four-state topology in Fig. 1(a).

Including the motional degrees of freedom, the many-body Hamiltonian

$$
\begin{aligned}
\hat{\mathcal{H}}= & \int \frac{d^{2} \mathbf{k}}{(2 \pi)^{2}} \sum_{j=1}^{N}\left\{\left[\frac{\hbar^{2}|\mathbf{k}|^{2}}{2 m}+(-1)^{j} \frac{\epsilon}{2}\right] \hat{\phi}_{j}^{\dagger}(\mathbf{k}) \hat{\phi}_{j}(\mathbf{k})\right. \\
& \left.-\frac{\Omega}{2}\left[e^{i \gamma_{j}} \hat{\phi}_{j+1}^{\dagger}\left(\mathbf{k}+\mathbf{k}_{j}\right) \hat{\phi}_{j}(\mathbf{k})+\text { H.c. }\right]\right\}
\end{aligned}
$$

describes a system of atoms with mass $m$ in $2 \mathrm{D}$ absent the ubiquitous confining potential. Here, $\left\{\phi_{j}^{\dagger}(\mathbf{k})\right\}$ is the spinor field operator describing the creation of an atom with momentum $\hbar \mathbf{k}$ in internal state $|j\rangle$; for even $N$, we introduce $\epsilon$, describing a detuning of alternating sign.

In what follows, we require that $\sum \mathbf{k}_{i}=0$, so that no momentum is transferred to an atom during a $|1\rangle \rightarrow \cdots \rightarrow$ $|N\rangle \rightarrow|1\rangle$ transition. We define the momenta exchange with differences $\mathbf{k}_{j}=\mathbf{K}_{j+1}-\mathbf{K}_{j}$ and require $\left\{\mathbf{K}_{j}\right\}$ to have a zero average. Moreover, $\bar{\gamma}$ may replace the phase $\gamma_{j}$ of each state vector $|j\rangle$, where $\bar{\gamma}=\sum_{i} \gamma_{i} / N$ [20], without loss of generality.

With the substitution $\hat{\varphi}_{j}^{\dagger}(\mathbf{q})=\hat{\phi}_{j}^{\dagger}\left(\mathbf{q}+\mathbf{K}_{j}\right)$, the Hamiltonian [Eq. (1)] separates into an integral $\int \sum_{j, j^{\prime}} \hat{\bar{\varphi}}_{j}^{\dagger}(\mathbf{q}) \bar{H}_{j, j^{\prime}}(\mathbf{q}) \bar{\varphi}_{j^{\prime}}(\mathbf{q}) d^{2} \mathbf{q} /(2 \pi)^{2}$ over $N \times N$ blocks,

$$
\begin{aligned}
\bar{H}_{j, j^{\prime}}(\mathbf{q})= & \frac{\hbar^{2}\left|\mathbf{q}+\mathbf{K}_{j}\right|^{2}}{2 m} \delta_{j, j^{\prime}}+(-1)^{j} \frac{\epsilon}{2} \delta_{j, j^{\prime}} \\
& -\frac{\Omega}{2}\left[e^{i \bar{\gamma}} \delta_{j-1, j^{\prime}}+\text { H.c. }\right],
\end{aligned}
$$


(a) Coupling scheme

(b) Geometry

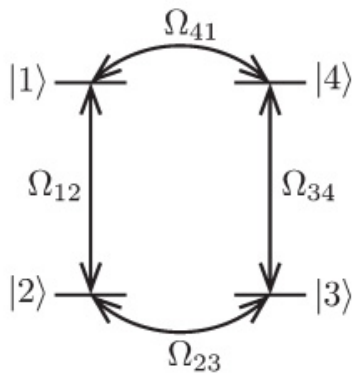

(c) Uncoupled dispersion

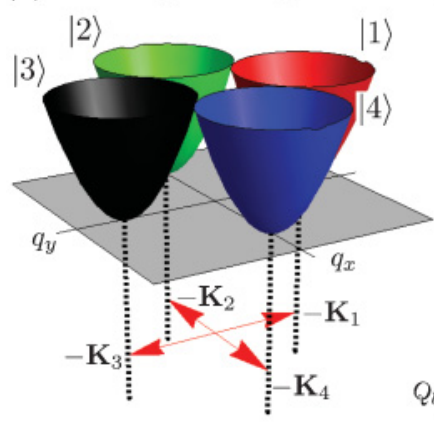

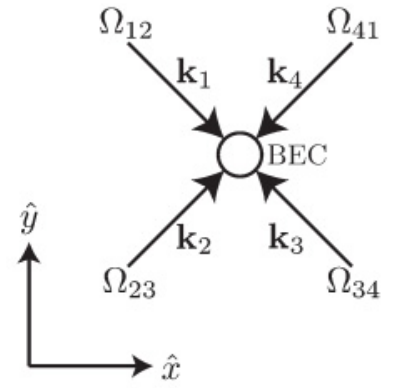

(d) Coupled dispersion

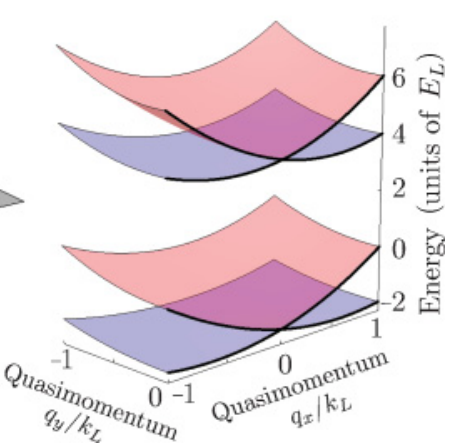

FIG. 1. (Color online) Four-level scheme. (a) Effective coupling between four internal ground or metastable atomic levels. (b) Spatial orientation of coupling fields. (c) Uncoupled eigenenergies for $\Omega=$ $\epsilon=0$. The four free parabolas are displaced by $\mathbf{K}_{j}$ in the $q_{x}-q_{y}$ plane. (d) Dispersion of four dressed states for $\Omega=3 E_{R}, \epsilon=0$, and $\bar{\gamma}=\pi / 4$, showing the expected Dirac points, one for each pair of dressed bands.

each labeled by a quasimomentum $\hbar \mathbf{q}$. The first (kinetic energy) term in Eq. (2) describes the 2D displaced parabolas depicted in Fig. 1(c). In analogy to band structure, the last (coupling) term in Eq. (2) has the form of an $N$ site 1D periodic tight-binding Hamiltonian with a "magnetic flux" $N \bar{\gamma}$ and a "hopping" matrix element $\Omega / 2$, where internal atomic states play the role of lattice sites. We diagonalize the coupling term (a zero-order approximation suitable when $\Omega$ is much larger than all other parameters) by transforming it into the basis conjugate to the spin-index $j$ with field operators

$$
\hat{\varphi}_{\ell}^{\dagger}(\mathbf{q})=\frac{1}{N^{1 / 2}} \sum_{j=1}^{N} e^{i 2 \pi \ell j / N} \hat{\varphi}_{j}^{\dagger}(\mathbf{q}) .
$$

The diagonalization provides the eigenenergies $E_{\ell}=$ $-\Omega \cos (2 \pi \ell / N-\bar{\gamma})$ of the coupling Hamiltonian, where $\ell \in$ $\{0, \ldots, N-1\}$ is analogous to the usual crystal momentum. The ground state can be made twofold degenerate by tuning $\bar{\gamma}$ to "magic" phases $\bar{\gamma}=2 \pi(p+1 / 2) / N$ for $p \in \mathbb{Z}$. The manufactured degeneracy between states at $\ell=0$ and 1 for $\bar{\gamma}=\pi / N$ is illustrated by Fig. 2 for $N=3$ and 4 .

When the displacement vectors $\mathbf{K}_{j}$ reside on the vertices of a regular polygon, $\mathbf{K}_{j}=-k_{L} \sin (2 \pi j / N) \mathbf{e}_{x}+$ $k_{L} \cos (2 \pi j / N) \mathbf{e}_{y}$, the full Hamiltonian matrix is

$$
\begin{aligned}
H_{\ell, \ell^{\prime}}(\mathbf{q})= & \left(\mathbf{q}^{2}+1+E_{\ell}\right) \delta_{\ell, \ell^{\prime}} \\
& +\left[\left(i q_{x}+q_{y}\right) \delta_{\ell-1, \ell^{\prime}}+\text { H.c. }\right]+\frac{\epsilon}{2} \delta_{\ell-N / 2, \ell^{\prime}},
\end{aligned}
$$

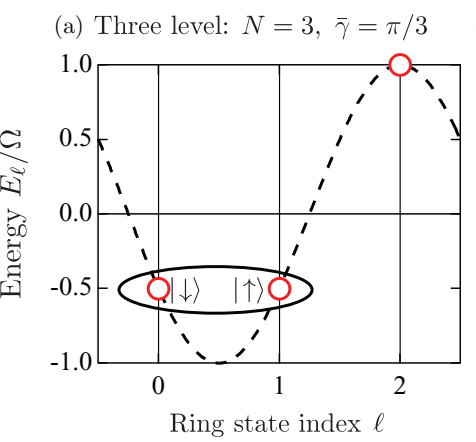

(b) Four level: $N=4, \bar{\gamma}=\pi / 4$

FIG. 2. (Color online) Eigenenergies $E_{\ell}$ of the coupling term in Eq. (2) showing the cosinusoidal energies evaluated at integer $\ell$. (a) Three-level case with $\bar{\gamma}=\pi / 3$ and (b) four-level case with $\bar{\gamma}=\pi / 4$. For both cases we identify pseudospin states $|\uparrow\rangle$ and $|\downarrow\rangle$ with the lowest energy pair of states.

where momenta and energies are expressed in recoil units, $k_{L}$ and $E_{L}=\hbar^{2} k_{L}^{2} / 2 m$, respectively. Assuming $\bar{\gamma} \approx \pi / N$, we focus on the manifold of two nearly degenerate states with $\ell=0$ and $\ell=1$, yielding the pseudospins $|\downarrow\rangle$ and $|\uparrow\rangle$ depicted in Fig. 2.

In what follows, we derive an effective $2 \times 2$ Hamiltonian $\check{H} \approx \breve{H}^{(0)}+\breve{H}^{(2)}+\breve{H}^{(3)}$ for this manifold up to third order in powers of $\Omega^{-1}$. In the subspace spanned by the lowest energy pseudospin-pair we obtain (up to a constant) a zeroorder Hamiltonian of the Rashba form

$$
\check{H}^{(0)}=|\mathbf{q}|^{2} \check{1}+\left(\check{\sigma}_{x} q_{y}-\check{\sigma}_{y} q_{x}\right)+\frac{\Delta_{Z}}{2} \check{\sigma}_{z},
$$

with a Zeeman field $\Delta_{Z}=E_{1}-E_{0} \approx-2 \Omega \bar{\gamma}^{\prime} \sin (\pi / N)$ generated by slight changes $\bar{\gamma}^{\prime}=\bar{\gamma}-\pi / N$ from the magic phase. At finite coupling $\Omega$, we adiabatically eliminate the excited states order-by-order in perturbation theory giving effective terms $H^{(n)}$ in the ground manifold Hamiltonian.

Since Eq. (3) is cyclic for $\epsilon=0$, we expect an energy shift at order $n=2$ in perturbation theory (effectively a Stark shift) and pseudospin-changing terms at order $n=N-1$. These terms serve to restore the $N$-fold rotational symmetry absent from Eq. (4) and in the analogous expressions of earlier proposals $[8,11,19]$. To understand the departure from the Rashba Hamiltonian, we first consider the simpler $\epsilon=0$ case.

For the $N=3$ case the second-order effective Hamiltonian

$$
\check{H}^{(2)}=-\frac{2}{3 \Omega}\left[|\mathbf{q}|^{2} \check{1}+2 \check{\sigma}_{x}\left(q_{y}^{2}-q_{x}^{2}\right)+\check{\sigma}_{y} q_{x} q_{y}\right]
$$

restores the expected threefold symmetry. For $N=4$, the second-order term acts as a state-independent Stark shift, but the third-order correction

$$
\check{H}^{(3)}=\frac{1}{2 \Omega^{2}}\left[\check{\sigma}_{x}\left(q_{y}^{3}-3 q_{y} q_{x}^{2}\right)-\check{\sigma}_{y}\left(q_{x}^{3}-3 q_{y}^{2} q_{x}\right)\right]
$$

restores the fourfold rotational symmetry. This term is reminiscent of the cubic Dresselhaus SO coupling present in GaAs 2D electron systems [3,21]. The Rashba Hamiltonian's [Eq. (4)] ground-state energy is minimized on the ring where $|\mathbf{q}|=1 / 2$; the perturbative terms modulate both the momenta and the energy where the minima occur [22]. Figure 3(a) shows this modulated energy for the four-level case at $\Omega=3 E_{L}$; Fig. 3(b) plots the peak-to-peak amplitude of the energy modulations. The perturbative analysis given by the dashed 
(a) 4-Level dispersion at $3 E_{L}$ coupling

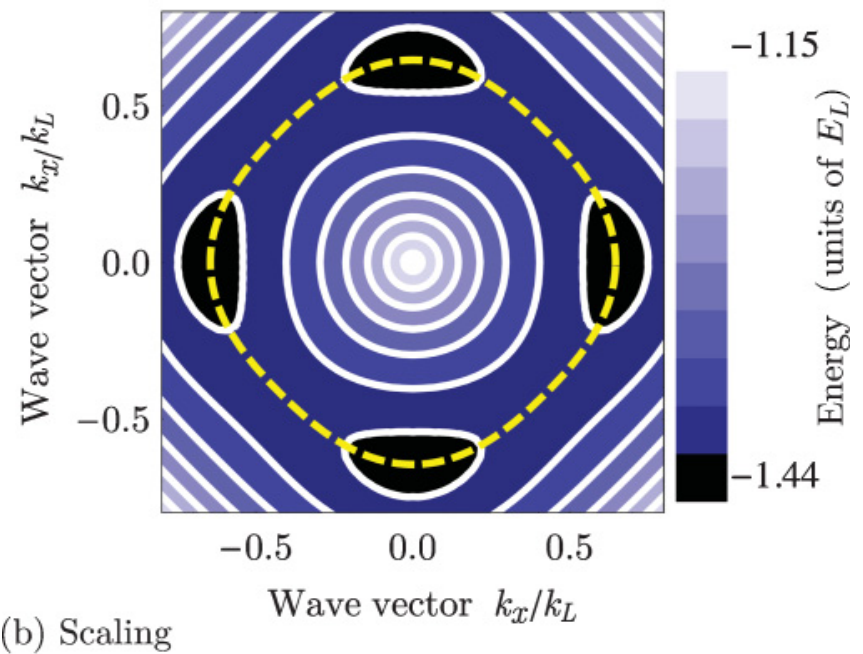

(b) Scaling

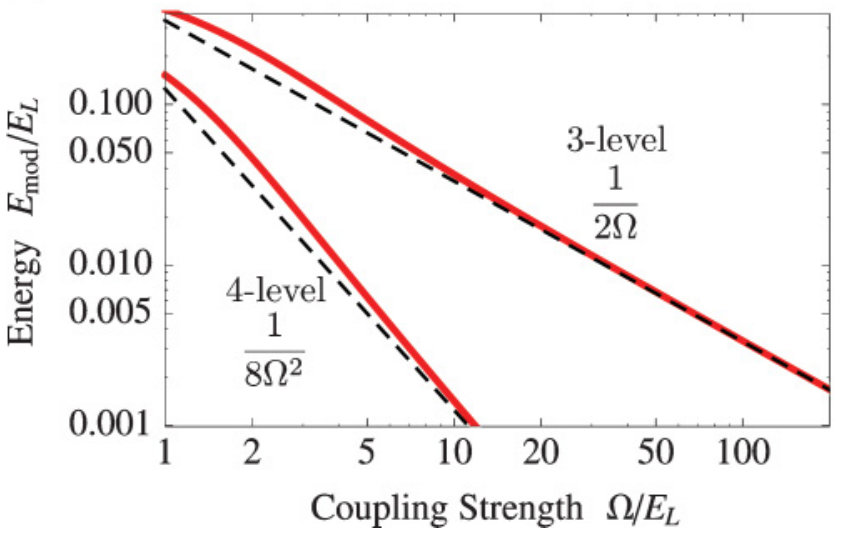

FIG. 3. (Color online) (a) Energy of the lowest eigenstate for $N=$ $4, \Omega=3 E_{L}, \epsilon=0$, and $\bar{\gamma}=\pi / 4$ plotted in the $q_{x}-q_{y}$ plane showing the fourfold rotational symmetry. The graph is colored according to the energy, and white curves mark contours of equal energy. The yellow dashed line depicts the azimuthal modulation of the radius in momentum of the energy minimum. (b) Peak-to-peak magnitude of the azimuthal energy modulations $E_{\text {mod }}$ plotted as a function of coupling $\Omega$, showing a rapid suppression for $N=4$ as compared to $N=3$. The solid red curves, the result of exactly solving the full Hamiltonian, quickly converge to the perturbation result (black dashed lines).

lines rapidly converges to the numerical solution to the full Hamiltonian given by the solid curves. Since the magnitude of the modulations scale like $\Omega^{2-N}$, the three-level case requires an impractically large $\Omega>100 E_{L}$ to reduce the corrections to the Rashba Hamiltonian below $10^{-3} E_{L}$, while the four-level case requires just $\Omega=10 E_{L}$. Interestingly, the familiar tripod scheme $[6,8,10,11,19]$ reduces to our $N=3$ ring model when far detuned from the excited state, with $\bar{\gamma}=0$ for red detuning and $\bar{\gamma}=\pi / 3$ for blue detuning. By contrast, the corrections to the Rashba model converge as $\Omega^{-2}$ in the standard resonant tripod (a four-level system).

The alternating detuning $\epsilon$ featured in Eq. (2) leads to a superlattice in the above-mentioned band-structure analogy, and its contribution can be included exactly. However, for a more painless description, we take $\epsilon / \Omega \ll 1$, which for $N=4$ adds a tunable Dresselhaus term $\check{H}_{D}=$ (a) Coupling diagram

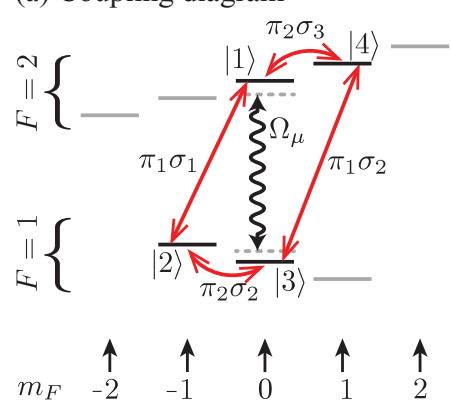

(b) Layout $\quad B_{0} \hat{y}$

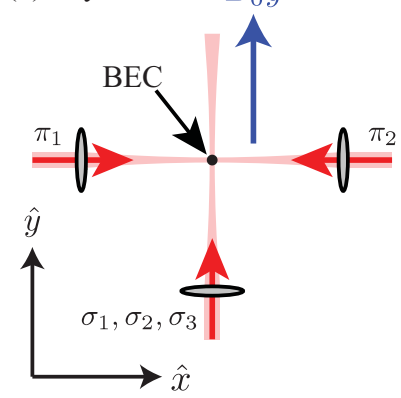

FIG. 4. (Color online) Four-level ring coupling scheme in ${ }^{87} \mathrm{Rb}$ involving hyperfine states $\left|F, m_{F}\right\rangle$ Raman-coupled by a total of five lasers marked $\sigma_{1}, \sigma_{2}, \sigma_{3}, \pi_{1}$, and $\pi_{2}$. (a) Level diagram: Each red curve represents a two-photon Raman transition with polarizations as marked by the field symbols. The ring states are mapped to physical states according to $|1\rangle=|2,0\rangle,|2\rangle=|1,-1\rangle,|3\rangle=|1,0\rangle$, and $|4\rangle=|2,1\rangle$. A $6.8-\mathrm{GHz}$ microwave field with coupling strength $\Omega_{\mu}$ ac Zeeman shifts the internal states $|1,0\rangle$ and $|2,0\rangle$. (b) Schematic layout: A bias magnetic field $B_{0} \approx 0.2 \mathrm{mT}$ lies along $\hat{y}$. Lasers $\pi_{1}$ and $\pi_{2}$ are $\pi$ polarized. Lasers $\sigma_{1}, \sigma_{2}$, and $\sigma_{3}$ are left circularly polarized and their relative phases define $N \bar{\gamma}=2 \phi_{\sigma_{2}}-\phi_{\sigma_{1}}-\phi_{\sigma_{3}}$.

$\left(E_{L} \epsilon / \sqrt{2} \Omega k_{L}\right)\left(\check{\sigma}_{x} q_{y}+\check{\sigma}_{y} q_{x}\right)$ at second order in $\Omega^{-1}$ (in original units). Thus, our scheme produces both Rashba and Dresselhaus couplings with strengths $\alpha=E_{L} / k_{L}$ and $\beta=E_{L} \epsilon / \sqrt{2} \Omega k_{L}$ along with a $\hat{z}$-aligned Zeeman field $\Delta_{Z} \approx$ $-\sqrt{2} \Omega \bar{\gamma}^{\prime}$. The laser configuration specifies $\alpha$, the alternating laser detunings set $\beta$, and $\Delta_{Z}$ is controlled by the average phase $\bar{\gamma}$. Together this allows for nearly complete control of the SO Hamiltonian.

Implementation. In the following, we specialize to a fourstate configuration within the $F=1$ and $F=2$ hyperfine manifolds of ${ }^{87} \mathrm{Rb}$ 's ${ }^{5} S_{1 / 2}$ ground electronic state [illustrated in Fig. 4(a)]. Each pair of states is Raman coupled by two lasers tuned between the ${ }^{5} P_{3 / 2}$ and ${ }^{5} P_{1 / 2}$ atomic excited manifolds $\left(E_{L} \approx h \times 2500 \mathrm{~Hz}\right)$. Although there are nominally eight transitions coupling four states we reuse three coupling lasers. The symbols $\sigma_{1}, \sigma_{2}, \sigma_{3}, \pi_{1}$, and $\pi_{2}$ each implicitly denote a laser frequency (that may be inferred from the level diagram in Fig. 4(a), the energy of each state $|j\rangle$, and the choice of two-photon detuning for one set of Raman lasers) and a direction given by Fig. 4(b). The first three symbols denote left circular polarization and their relative phases define $N \bar{\gamma}=2 \phi_{\sigma_{2}}-\phi_{\sigma_{1}}-\phi_{\sigma_{3}}$. Lasers $\pi_{1}$ and $\pi_{2}$ are linearly polarized along the magnetic field and produce canceling phases in the Hamiltonian and thus do not contribute to the phase sum. Additionally, state labels will match those of the paper given the ordering of frequency $v_{\pi_{1}}>v_{\sigma_{3}}>$ $v_{\pi_{2}}>v_{\sigma_{2}}>v_{\sigma_{1}}$. A bias field along $\hat{y}$ and an ac Zeeman shift provided by a microwave field along $\hat{x}$ produce a composite Zeeman shift that spectroscopically isolates [13] the hyperfine changing transitions by at least $10 \Omega$. In addition, $|2\rangle$ and 14) shift together with an applied magnetic field, allowing straightforward control of the detuning $\epsilon$, thus contributing Dresselhaus coupling.

In practice, electronic equipment will introduce unwanted time-varying terms into the Hamiltonian. However, the effects of magnetic field detuning $\Delta \epsilon$, laser intensity shifts $\Delta \Omega$ 
and phase shifts $\Delta \bar{\gamma}$ can have negligible contributions in typical laboratory settings. In the scheme shown in Fig. 4, time-varying magnetic fields induce identical linear Zeeman shifts of $|2\rangle$ and $|4\rangle$ that cancel at lowest order in perturbation theory (while $|1\rangle$ and $|3\rangle$ are first-order insensitive to magnetic fields). These Zeeman shifts give an additional Dresselhaus contribution $\check{H}_{\Delta D} \propto \Delta \epsilon\left(\check{\sigma}_{x} q_{y}+\check{\sigma}_{y} q_{x}\right)$; near the minima of the Rashba Hamiltonian, the energy shift from the resulting Dresselhaus term would be $0.0005 E_{L}$ for our $\Delta \epsilon \approx h \times 50 \mathrm{~Hz}$ detuning noise amplitude [13].

Shifts (resulting from laser intensity noise) in a single $\Omega_{j, j+1}$ matrix element add coupling terms $\check{H}_{\Delta \Omega} \approx$ $-\sqrt{2} \Delta \Omega_{j, j+1}\left(\boldsymbol{K}_{\boldsymbol{j}}+\boldsymbol{K}_{\boldsymbol{j}+\mathbf{1}}\right) \cdot \check{\boldsymbol{\sigma}} / 8 k_{L}$, where $\check{\boldsymbol{\sigma}}$ is the vector of Pauli matrices. For lasers stabilized at the $0.1 \%$ level, and with $\Omega=10 E_{L}$, the unwanted coupling terms have magnitude $0.002 E_{L}$.

Phase shifts $\Delta \bar{\gamma}$ directly modulate the effective Zeeman fields $\check{H}_{\Delta \phi} \approx-\sqrt{2} \Omega \Delta \bar{\gamma} \check{\sigma}_{z} / 2$, which opens a gap at the Dirac point. For ${ }^{87} \mathrm{Rb}$, we require the resulting gap energy to be less than a typical $T=10 \mathrm{nK}$ temperature. For $\Omega=10 E_{L}$, the rms phase noise must have an amplitude $\Delta \bar{\gamma}<0.04$ rad. Even for independent lasers, this level of phase stability is routine [24].

Discussion. Inspection of Eq. (3) shows that Dirac points are present for all $\Omega$ and $\epsilon$ provided $\bar{\gamma}=\pi / N$, even when perturbative corrections are important. As a result, properties of fermion systems which depend only on the topology of the dressed-state dispersion may be insensitive to small corrections to Eq. (3). In contrast, bosons generally condense at the energy minima. Thus, for insufficient laser coupling, local minima may spoil correlation physics potentially arising from the Rashba Hamiltonian's degenerate ring of minima $[19,23]$.

The proposed coupling scheme provides a robust platform for generating SO coupling for neutral atoms. Because the two spin states are the lowest energy dressed states, atomatom interactions cannot induce collisional decay $[14,15]$. In addition, this technique can require considerably less laser intensity than prior far-detuned schemes to reach nearly pure SO coupled Hamiltonians, greatly reducing spontaneous emission. Lastly our specific implementation uses only the $\delta m_{F}=0, \pm 1$ Raman transitions allowed at large atomic detuning in the alkali-metal atoms $[6,11]$.

We thank KITP Santa Barbara (NSF Grant No. PHY0551164) where this collaboration was initiated. D.L.C. and I.B.S. acknowledge the financial support of the NSF through the PFC at JQI and of the ARO with funds from both the Atomtronics MURI and the DARPA OLE Program. G.J acknowledges support from the EU project STREP NAMEQUAM.
[1] C. L. Kane and E. J. Mele, Phys. Rev. Lett. 95, 146802 (2005).

[2] M. Z. Hasan and C. L. Kane, Rev. Mod. Phys. 82, 3045 (2010).

[3] J. D. Koralek, C. P. Weber, J. Orenstein, B. A. Bernevig, S.-C. Zhang, S. Mack, and D. D. Awschalom, Nature (London) 458, 610 (2009).

[4] A. P. Schnyder, S. Ryu, A. Furusaki, and A. W. W. Ludwig, Phys. Rev. B 78, 195125 (2008).

[5] J. D. Sau, S. Tewari, R. M. Lutchyn, T. D. Stanescu, and S. Das Sarma, Phys. Rev. B 82, 214509 (2010).

[6] J. Dalibard, F. Gerbier, G. Juzeliūnas, and P. Öhberg, e-print arXiv:1008.5378 (accepted to Rev. Mod. Phys.).

[7] K. Osterloh, M. Baig, L. Santos, P. Zoller, and M. Lewenstein, Phys. Rev. Lett. 95, 010403 (2005).

[8] J. Ruseckas, G. Juzeliūnas, P. Öhberg, and M. Fleischhauer, Phys. Rev. Lett. 95, 010404 (2005).

[9] S.-L. Zhu, H. Fu, C.-J. Wu, S.-C. Zhang, and L.-M. Duan, Phys. Rev. Lett. 97, 240401 (2006).

[10] X.-J. Liu, M. F. Borunda, X. Liu, and J. Sinova, Phys. Rev. Lett. 102, 046402 (2009).

[11] G. Juzeliunas, J. Ruseckas, and J. Dalibard, Phys. Rev. A 81, 053403 (2010).

[12] N. Goldman, I. Satija, P. Nikolic, A. Bermudez, M. A. MartinDelgado, M. Lewenstein, and I. B. Spielman, Phys. Rev. Lett. 105, 255302 (2010).
[13] Y. J. Lin, K. Jimenez-Garcia, and I. B. Spielman, Nature (London) 471, 83 (2011).

[14] I. B. Spielman, P. R. Johnson, J. H. Huckans, C. D. Fertig, S. L. Rolston, W. D. Phillips, and J. V. Porto, Phys. Rev. A 73, 020702(R) (2006).

[15] R. A. Williams, L. J. LeBlanc, M. Beeler, K. Jiménez-García, and I. B. Spielman (unpublished). In these systems, we observe excited state lifetimes around $20 \mathrm{~ms}$.

[16] Reimplementing the tripod or tetrapod schemes all within the electronic ground state is also possible $[6,11]$.

[17] G. Dresselhaus, Phys. Rev. 100, 580 (1955).

[18] Y. A. Bychkov and E. I. Rashba, J. Phys. C 17, 6039 (1984).

[19] T. D. Stanescu, B. Anderson, and V. Galitski, Phys. Rev. A 78, 023616 (2008).

[20] The displacement vectors $\mathbf{K}_{j}=\sum_{l} l \mathbf{k}_{l+j-1} / N$ and phases $\theta_{j}=$ $\sum_{l=1}^{j-1}\left(\gamma_{l}-\bar{\gamma}\right)$ define these transformations explicitly.

[21] T. D. Stanescu and V. Galitski, Phys. Rev. B 75, 125307 (2007).

[22] In practice, the confining harmonic potential also lifts the Rashba Hamiltonian's ground state degeneracy at the level of the typical $\approx 10 \mathrm{~Hz}$ trap frequencies [23]. Thus we consider perturbative corrections below $0.001 E_{L} \approx h \times 2.5 \mathrm{~Hz}$ as negligible.

[23] J. Larson and E. Sjöqvist, Phys. Rev. A 79, 043627 (2009).

[24] L. Cacciapuoti, M. de Angelis, M. Fattori, G. Lamporesi, T. Petelski, M. Prevedelli, J. Stuhler, and G. M. Tino, Rev. Sci. Instrum. 76, 053111 (2005). 